\title{
The Treatment and Prognosis of Refractory and Super-Refractory Status Epilepticus
}

\section{(ㄷ) (i) (우)}

Authors

Stephanie Gollwitzer, Hajo M. Hamer

\author{
Affiliation \\ Epilepsy Center, Department of Neurology, University \\ Hospital of Erlangen, Erlangen, Germany
}

Key words

status epilepticus, refractory status epilepticus, superrefractory status epilepticus

\author{
Bibliography \\ DOI https://doi.org/10.1055/s-0043-110165 \\ ISSN 2511-1795 \\ Correspondence \\ Dr. med. Stephanie Gollwitzer \\ Klinik für Neurologie \\ Epilepsiezentrum \\ Universitätsklinikum Erlangen \\ Schwabachanlage 6 \\ 91054 Erlangen \\ Germany \\ stephanie.gollwitzer@uk-erlangen.de
}

Neurology International Open 2017; 1: E204-E210

(c) Georg Thieme Verlag KG Stuttgart · New York

\begin{abstract}
Refractory status epilepticus (RSE) is defined as status epilepticus (SE) persisting over 60 min and resistant to treatment with benzodiazepines and non-sedating antiepileptic drugs. The term super-refractory status epilepticus (SRSE) refers to a refractory episode continuing under general anesthesia for more than $24 \mathrm{~h}$. RSE is treated with a combination of non-sedating AED and i.v. anesthetics; first choice drugs are midazolam, propofol and thiopental. The management of super-refractory status epilepticus (SRSE) is challenging as clear evidence-based guidelines are lacking. Recommendations are mainly based on case reports and small case series. Therapeutic options include ketamine, inhalational anesthetics, steroids and immunoglobulins. Ketogenic diet, electroconvulsive therapy and epilepsy surgery are also considered as potentially effective. A promising new approach is the neurosteroid allopregnanolone. Mortality of RSE and SRSE is largely influenced by the etiology and is markedly higher as compared to non-refractory status epilepticus. It was reported to be about $30 \%$ and $50 \%$, respectively.
\end{abstract}

\section{Introduction}

Status epilepticus (SE) is one of the most common emergencies in neurocritical care. In Germany, the incidence of SE is about 17/100 000 per year, increasing with age [1]. Especially generalized convulsive (tonic-clonic) status epilepticus (GCSE) is still associated with a high risk of morbidity and mortality [2].

SE is defined as a single prolonged seizure or a series of two or more consecutive seizures without return to neurological baseline between seizures. According to the most recent definition, a generalized convulsive status epilepticus is already diagnosed after a minimum seizure duration of five minutes, because the likelihood of spontaneous termination decreases after this time period; consequently, treatment should be initiated without further delay [3]. With significantly less experimental data on focal SE and absence SE available, the reported minimum seizure duration for the two conditions is $10 \mathrm{~min}$ and $10-15 \mathrm{~min}$, respectively, reflecting the assumption that neuronal damage is to be expected at a later point in time or to a lesser degree with focal SE and absence SE compared to GCSE [3]. However, already after 5 min the likelihood that a seizure will terminate spontaneously is low, regardless of the type of SE [4].

Three clinical stages with different treatment regimens are differentiated: 1 . The initial phase: seizure duration $\leq 10 \mathrm{~min}$ in a previously untreated patient; treatment with benzodiazepines (lorazepam, midazolam, clonazepam). 2. Established SE: SE persisting for 10-30 (max. 60) min. Besides benzodiazepines, non-sedating IV anticonvulsants (phenytoin, valproic acid, levetiracetam) can be used. 3. Refractory SE: The status epilepticus has already continued for $60 \mathrm{~min}$, despite appropriately dosed anticonvulsant treatment. Refractory GCSE is an acute life-threatening situation, requiring fast initiation of intensive-care monitoring with preparedness for endotracheal intubation and treatment escalation to IV anesthetic administration. 4. If SE continues after $24 \mathrm{~h}$ of treatment with anesthetic agents, it is termed super-refractory SE. With no 
systematic data on treatment available, recommendations are largely based on case reports [5].

Especially refractory and super-refractory SE are associated with significantly increased morbidity and mortality and continue to be very challenging conditions to treat. Therefore, the aim of this review was to provide an overview of the current treatment options and their prognoses.

\section{The Refractory Status Epilepticus (RSE) Incidence}

RSE incidence rates are mostly estimated, using data from retrospective studies. They are reported to be $31 \%$ to $43 \%$ of all patients with SE treated in intensive care units [6-8]. A recent prospective analysis found that $33 \%$ of SE cases were refractory in nature [2]. It is assumed that RSE more commonly develops in patients with acute brain damage than in patients with pre-existing epilepsy $[7,9]$. However, RSE may also occur in previously healthy adults as the first manifestation of epilepsy ('de novo' status epilepticus) [10].

\section{Management}

The aggressiveness of treatment is chosen according to the type of SE.

In patients with GCSE, it is vital to urgently terminate the seizure state, as otherwise there is a risk of neuronal death due to massive glutamatergic receptor activity with increased calcium influx into cells, ultimately leading to life-threatening apoptosis and necrosis [11-12].

Patients experiencing refractory generalized convulsive status epilepticus require treatment in an intensive care unit, ideally in a neurocritical care unit with EEG monitoring facilities. In cases where the cause of SE can be identified, this should be addressed and appropriate treatment provided.

Since at this point in time, benzodiazepines and non-sedating anticonvulsants have already been used unsuccessfully, additional fast administration of IV anesthetics is the basic principle in the treatment RSE with generalized tonic-clonic seizures. In the majority of cases, this approach is successful and terminates SE, but up to $10 \%$ of patients experience renewed seizures during the course of this treatment or with dose reduction of the anesthetic agents [11]. First-line treatment options include midazolam, propofol and thiopental. With no comparative studies available, no evidence-based superiority of any of the available drugs can be postulated; therefore, the choice of treatment should be made on an individual basis and take into account the respective drug interaction and adverse drug reaction profiles [13-16].

The use of thiopental for the treatment of SE is backed by many years of experience; this agent has a strong anticonvulsant effect [17]. However, patients may experience significant hypotension under this treatment, frequently requiring additional vasopressor administration. Other factors to be taken into consideration include the comparatively longer half-life of thiopental-increasing the risk of accumulation and frequently prolonging mechanical ventilation times-, its drug interaction potential, immunosuppression, and potential hepatic and pancreatic toxicity [18]. Treatment is typically started with the administration of an initial bolus of $5 \mathrm{mg} / \mathrm{kg}$ body weight; the maintenance dose is $3-7 \mathrm{mg} / \mathrm{kg}$ BW/h for $24 \mathrm{~h}$ under EEG monitoring. The therapeutic end point may for instance be burst-suppression pattern in the EEG for $24 \mathrm{~h}$. Unfortunately, no conclusive data is available regarding the optimum duration of the burst-suppression pattern before the sedation can be reduced.

Propofol has favorable pharmacokinetic properties, including its very rapid onset of action and the fast recovery even after prolonged administration. Thus, major drug-drug interactions are unlikely. Patients treated with propofol may experience a relevant drop in blood pressure, too. After administration over several days, the risk of propofol-infusion syndrome should be taken into consideration, a rare but in the majority of cases fatal complication [19]. Lactic acidosis, rhabdomyolysis, hyperkalemia, and cardiac dysfunction are usually present. Propofol is also administered, using EEG guidance, to achieve a burst-suppression pattern; treatment is started with an initial bolus of $2 \mathrm{mg} / \mathrm{kg}$ body weight and maintained with approx. 4-10 mg/kg BW/h [20].

As a short-acting benzodiazepine with a strong anticonvulsant effect, midazolam is also suitable for continuous administration. However, in contrast to the other anesthetic agents, midazolam does not normally achieve a burst-suppression pattern in the EEG, even if administered in high doses. Midazolam may have a cardiodepressive effect. In addition, patients may rapidly develop tolerance to midazolam which in turn promotes seizure recurrence [21]. It is recommended to start treatment with an initial bolus of $0.2 \mathrm{mg} / \mathrm{kg}$ body weight and maintain it with the continuous administration of $0.1-0.5 \mathrm{mg} / \mathrm{kg} \mathrm{BW} / \mathrm{h}$.

When deciding upon the treatment of refractory nonconvulsive $\mathrm{SE}$, it is also necessary to balance potential negative treatment effects against the inherent morbidity and mortality of this condition. Nonconvulsive SE (NCSE) is used as an umbrella term incorporating various entities, including NCSE with or without coma, absence SE, aura SE, aphasic SE, or autonomous SE [3]. Establishing the type of status epilepticus on an individual basis is a critical prerequisite for the choice of the therapeutic approach, even though controversy remains-and this applies to all types of NCSEwith respect to whether, and to what extent, clinically relevant neuronal injury occurs and whether urgent and aggressive termination of SE, accepting treatment-associated risks, should be the primary goal. In fact, it appears that the functional outcome significantly depends on etiological factors and the type of seizure activity; thus, treatment should be tailored to the individual patient's situation $[22,23]$. Several recent retrospective studies found that, especially in patients with milder types of SE, aggressive treatment with anesthetic agents may be associated with higher mortality, higher complication rates, and longer hospital stays [24-26]. The most important point of criticism regarding these retrospective cohorts is that inherent differences between the groups may still have been present despite the authors' significant efforts to adjust for confounders. It is challenging to exclude with certainty that more severely affected patients received anesthetic agents more frequently or in different doses by the treating physicians than less severe cases [16]. This underscores the urgent need for randomized and prospective studies on the management of status epilepticus to prevent that disputable results are uncritical integrated in clinical treatment pathways. 


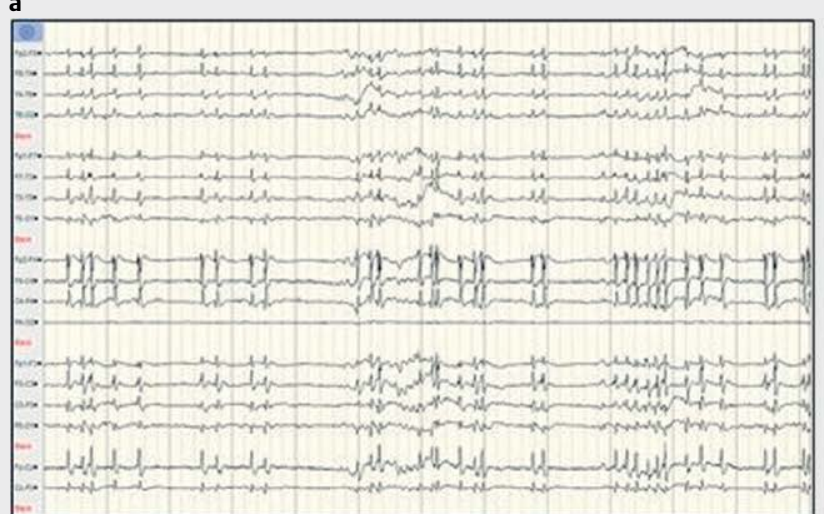

c

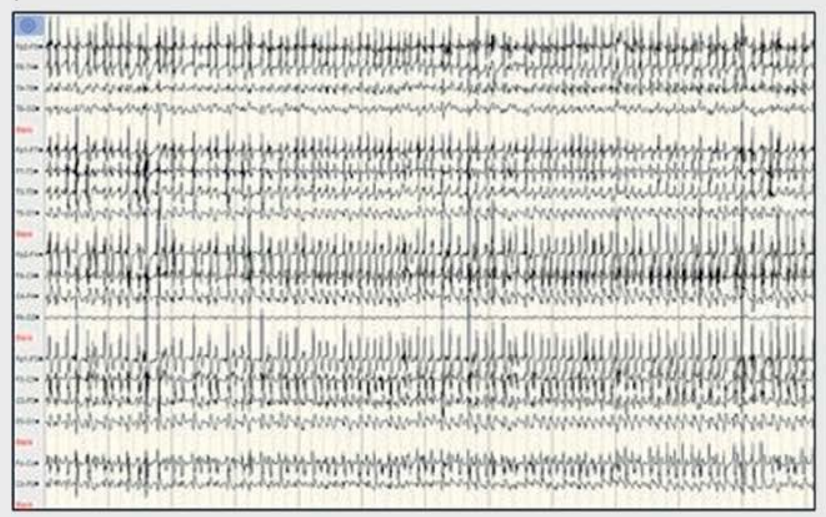

b

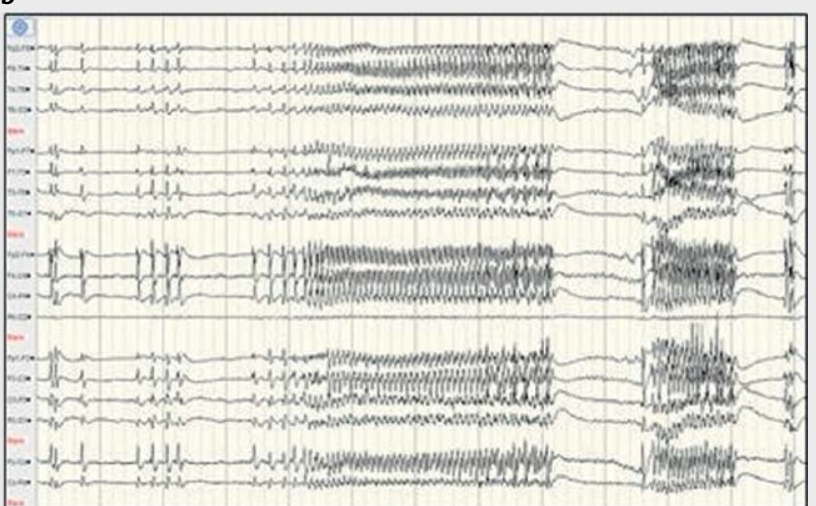

d

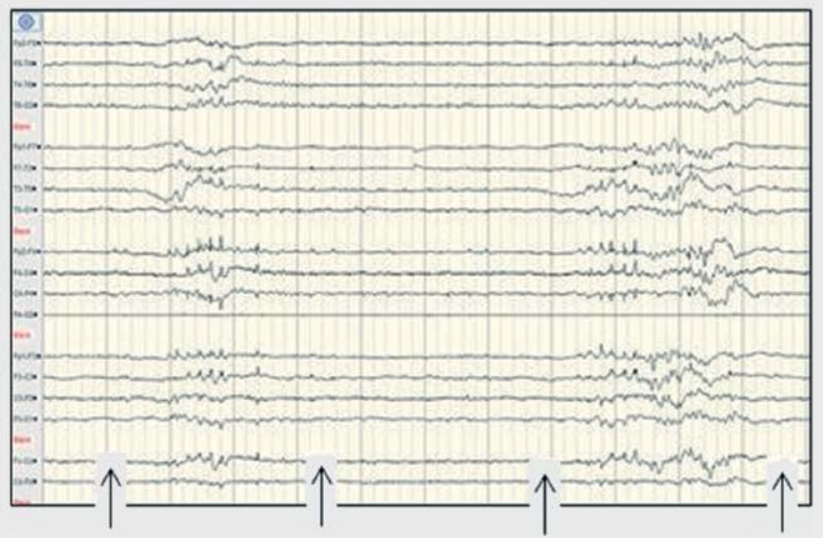

- Fig. 1 EEG of a 34-year-old patient with suspected antibody-negative autoimmune encephalitis and nonconvulsive SRSE of 6 days' duration, treated with valproate, levetiracetam, phenytoin, lacosamide, and ultimately propofol and thiopental. Continuous EEG monitoring showed periods of rhythmic epileptic activity a with a frequency $>2.5 \mathrm{~Hz}$ and intermittent seizure patterns with evolution in frequency and morphology b, c. Under IV anesthesia, a burst-suppression pattern (arrows) was achieved $\mathbf{d}$.

Continuous EEG monitoring can aid diagnosis of refractory status epilepticus and greatly assist in monitoring treatment response ( $\vee$ Fig. 1). It can be assumed that nonconvulsive SE in patients with impaired consciousness, particularly in those with underlying central nervous system disease, continues to be underdiagnosed and that the actual incidence is higher than reported so far [27, 28]. In case continuous EEG monitoring is not feasible, frequent EEG recordings are indispensable to guide treatment.

\section{The Super-refractory Status Epilepticus (SRSE)}

\section{Incidence}

Introduced during the London-Innsbruck Colloquium on status epilepticus in 2011, the term "super-refractory status epilepticus" refers to SE of more than $24 \mathrm{~h}$ duration despite appropriately dosed treatment with anesthetic agents [5]. According to more recent prospective studies, the incidence of SRSE is approximately $4 \%$ to $9 \%$ of SE cases $[2,9]$. SRSE cases were more frequently observed in younger patients with severely impaired consciousness at initial presentation [2]. Autoimmune inflammatory conditions are reported to be the most common cause of SRSE [29-30].

\section{Management}

The recommendations for the treatment of SRSE are primarily based on case reports; data from randomized trials are missing. If the underlying condition, such as autoimmune or infectious encephalitis or intoxication, can be identified, treating this condition has a strong anticonvulsant effect in patients with SRSE [31]. As with the treatment of RSE, the use of IV anesthetic agents is the foundation of symptomatic treatment in SRSE patients.

In patients who do not respond to the medications mentioned above, an attempt with ketamine in combination with benzodiazepines can be made. In contrast to other anesthetic agents, ketamine does not bind to the GABA-A receptors, but acts as an antagonist on NMDA receptors. Since in the course of SE, GABA receptors are internalized and NMDA receptors upregulated, increased efficacy of ketamine can be assumed [32,33]. Another advantage of this medication is that it does not adversely affect cardiac function, helping to reduce the need for additional vasopressors. A retrospective multicenter study analyzing 58 cases reported termina- 
tion of SE along with additional ketamine administration in $32 \%$ of cases [34].

Single-case reports indicate that inhalation anesthetic agents may be effective as well. In a case series, desflurane and isoflurane terminated SE in 4 of 7 cases; however, under this treatment complications, including one mesenteric artery infarction, one deep venous thrombosis, and one hemorrhagic leukoencephalopathy, occurred [35]. Overall, the use of inhalation anesthetic agents seems to be limited by the high treatment costs and the high complication rates.

As in all other phases of SE, anticonvulsants are used to treat SRSE as well. Especially during anesthesia and after discontinuation of anesthetics, effective adjunctive therapy with anticonvulsants is critical. Systematic data indicating which drugs or drug combinations should be preferentially used are not available. Suitable medications include anticonvulsants which can initially be administered intravenously or via nasogastric tube or percutaneous endoscopic gastrostomy, ensuring that therapeutic blood levels are rapidly attained. Levetiracetam, lacosamide, carbamazepine, perampanel, phenobarbital, phenytoin, topiramate, and valproate are widely used. However, only phenobarbital, phenytoin and valproate are approved for the treatment of SE, while the remaining anticonvulsants are not explicitly approved for this indication. It is important to ensure adequate dosing and fast uptitration. Combinations of more than 3 non-sedating anticonvulsants should be avoided. In case a drug shows no effect, it can usually be discontinued and replaced by another agent, even in the acute phase. In addition, continuous magnesium infusion can be attempted.

Steroids have been used in the treatment of SE for a number of years. There are two reasons supporting the use of steroids to treat SRSE: First, autoimmune encephalitis is presumably the most common cause of SRSE, even if patients do not test positive for antibodies. In this case, immunomodulatory therapy would be a causative treatment. Second, inflammatory processes such as the activation of pro-inflammatory cytokines, e. g. interleukin- $1 \beta$ and TNF- $\alpha$, are likely to play an important role in epileptogenesis/ictogenesis; consequently, anti-inflammatory therapy may have a significant anti-seizure potential [36]. Besides steroids, the administration of immunoglobulins and the use of plasmapheresis could be considered, although the currently available data are not sufficient to support a general recommendation in this respect [37].

Apart from pharmacological treatment strategies, ketogenic diet (KD) has been reported to be an effective treatment for SRSE in various case studies, especially in pediatric patients, but less frequently in adult patients too [38, 39]. An almost carbohydrate-free diet has been used to treat epilepsy since the 1920s. One of the largest sample of adult SRSE patients treated with KD comprised 10 patients; 9 of these patients experienced termination of SRSE under KD. The median time from initiation of KD to termination of SRSE was 3 days. In 2 cases, SE was only terminated after 12 and 31 days, respectively; whether there was a causal relationship remains unproven in these cases. In all patients, ketosis was achieved without problems and KD was not associated with any significant complications [39]. In an ICU, KD can easily be initiated intravenously and continued enterally, if needed [40]. Commercial products typically contain fat and carbohydrates/proteins in a ratio of $4: 1$ or 3:1. Achievement and maintenance of ketosis is verified by measuring the levels of ketone bodies in serum and/or urine. Contraindications for KD include various metabolic defects, such as pyruvate decarboxylase deficiency, fatty acid oxidation disorders and gluconeogenesis disorders. Concomitant administration of topiramate or zonisamide with KD increases the risk of metabolic acidosis. Valproate inhibits hepatic beta-oxidation, potentially making it more difficult to achieve ketosis.

Another non-pharmacological intervention to treat SRSE is therapeutic hypothermia [41]. Hypothermia reduces cerebral oxygen and ATP consumption, inhibits glutamate release and lowers oxidative stress. In addition, hypothermia exerts an anti-edematous effect, resulting from the reduced permeability of the blood brain barrier, and an anti-inflammatory effect. Because of these mechanisms, hypothermia is thought to have both an anticonvulsant and a neuroprotective effect $[42,43]$. Recently, the randomized, multicenter HYBERNATUS study systematically evaluated the effect of hypothermia for the treatment of SE on the outcome after 90 days. Altogether, 270 patients were included in the study. However, treatment with hypothermia was not associated with a more favorable outcome compared with standard treatment. Moreover, more complications occurred in the hypothermia group compared with the control group [41].

Emergency epilepsy surgery may be considered in selected cases if neuroimaging studies clearly identify a distinct lesion as the cause of SRSE or if a focal, circumscribed epileptogenic zone can be defined electroclinically ( $\triangleright$ Fig. 2). Focal resection to treat patients with, for instance, focal cortical dysplasia or tumor seems to be a sensible approach. In patients with Rasmussen's encephalitis, hemispherectomy is often the last treatment option to terminate epilepsia partialis continua. For palliative intervention, corpus callosotomy may be considered in individual cases [44]. It remains unclear at what point in time over the course of SRSE a surgical intervention should be performed.

In individual cases, electroconvulsive therapy (ECT) was successfully used to treat SRSE $[45,46]$. Here it may be necessary to reduce the doses of the anticonvulsants and anesthetics the patient is receiving to facilitate seizure induction by ECT. Approximately 5-8 ECT treatment sessions on consecutive days are recommended.

Recently, the neurosteroid allopregnanolone was presented as another promising treatment option for SRSE [47]. Over the course of SRSE, the effect of GABAergic substances progressively weakens due to the internalization of synaptic GABA-A receptors. In an animal model, allopregnanolone modulated GABA-A receptors and reduced their internalization [48]. So far, clinical experience with the use of allopregnanolone in humans is limited. In two pediatric patients, treatment with allopregnanolone in combination with GABAergic drugs successfully terminated SE [47]. Currently, a randomized, multicenter, double-blind study on the treatment of SE with allopregnanolone is being performed [49].

\section{The Prognosis and Mortality of Refractory and Super-refractory SE}

The mortality rates of RSE and SRSE range from $15 \%$ to $54 \%$ and thus exceed the mortality of non-refractory SE (11-37\%) by far. In the most recent study, $24.5 \%$ of patients with RSE, $37.9 \%$ of pa- 

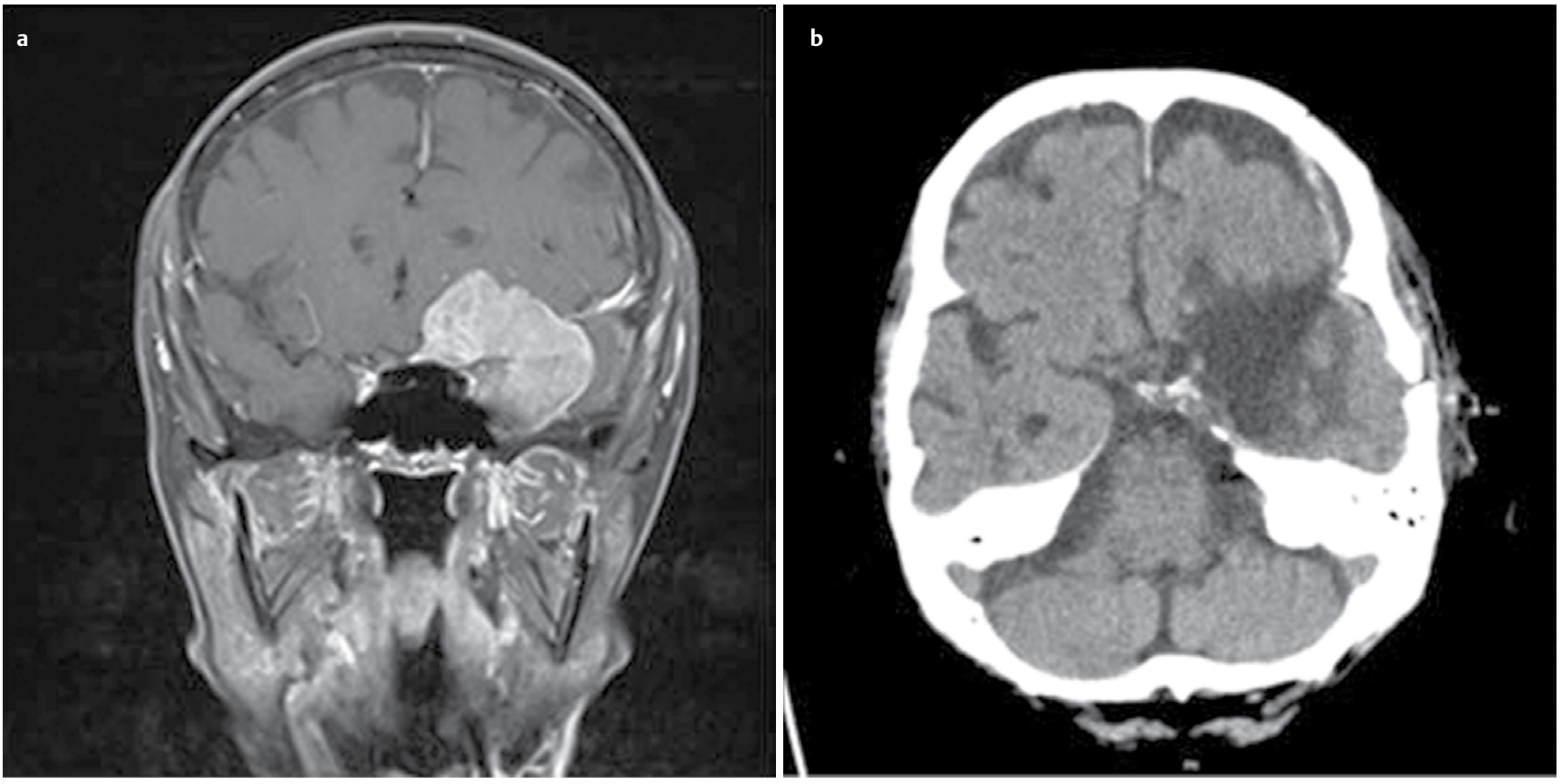

- Fig. 2 81-year-old patient with sphenoid wing meningioma and nonconvulsive SRSE left temporally a of 4-week duration, last treated with levetiracetam, phenytoin, lacosamide, midazolam, and propofol. In this situation, it was decided to perform "SE-surgical" resection of the meningioma and anterior parts of the temporal lobe $\mathbf{b}$. Postoperative improvement of the EEG findings. Termination of SRSE only after another change in the combination of anticonvulsants and addition of perampanel.

tients with SRSE, but only $9.8 \%$ of patients with non-refractory SE died [2]. The high variability of the reported mortality rates is explained by the significant heterogeneity of the patient populations studied. Clinical scores are used to assess functional outcome and mortality in patients with SE. For instance, the Status epilepticus Outcome Score (STESS) is a valid and easy-to-use scoring system to predict mortality. Factors included in SE-related scores are consciousness, type of seizure, age, and the presence of epilepsy. Using these four variables, the mortality risk can be predicted with high sensitivity and relatively good specificity [52]. In addition, the epidemiology-based mortality score in status epilepticus (EMSE) is available as another scoring system, taking into account the etiology of SE, patients' age, comorbidities, and EEG findings [53].

The underlying etiology of SE certainly as a significant impact on functional outcome [50,51]. Especially patients developing (S) RSE after hypoxic brain injury have per se a poor prognoses. Since hypoxic encephalopathies with generalized periodic discharges in the EEG tend to be highly refractory to treatment and have a very poor prognosis, many working groups consider them as entities of their own, not meeting the strict definition of SE [54, 55]. By contrast, factors such as younger age, known epilepsy and initially (at least partially) maintained consciousness are indicative of a better outcome $[50,56]$. Increasing duration of status epilepticus indicates a poorer prognosis; however, even after prolonged therapeutic coma and status epilepticus persisting over several weeks, there is a chance of achieving a good functional outcome, as long as complications can be avoided [57-59]. Thus, therapeutic nihilism is not indicated.

\section{Conclusion}

Refractory and super-refractory status epilepticus represent neurological emergencies, requiring treatment under intensive EEG monitoring in a neurocritical care unit. Beyond the administration of non-sedating anticonvulsants, refractory status epilepticus is treated with intravenous (IV) anesthetics. Here, preferred drugs include midazolam, propofol and thiopental. With only limited data from studies available, established clinical pathways for patients with super-refractory status epilepticus are non-existent. Treatment options include ketamine, inhalation anesthetics, immunomodulation, ketogenic diet, hypothermia, electroconvulsive therapy, and, in selected cases, epilepsy surgery.

Refractory and super-refractory status epilepticus are associated with significant morbidity and mortality. Nonetheless, complete recovery without residual neurological deficits has been observed even after prolonged episodes of status epilepticus.

\section{Conflict of interest}

S. Gollwitzer received lecture fees and/or support for CME events from Desitin, Eisai and UCB Pharma. In addition, she received consulting fees from UCB Pharma. H. M. Hamer received lecture or manuscript fees and/or support for continuing medical education (CME) events from Ad-Tech, Desitin, Eisai, GSK, IQWiG, Hexal, Ingelheim Boehringer, NeuroConsil, Nihon Kohden, Novartis, Pfizer, Thieme, and UCB Pharma as well as from the universities of Essen, 
Hamburg, Hannover, Marburg, Munich, and Saarbrücken. Furthermore, he received consulting fees from Cerbomed, Desitin, Eisai, GSK, Pfizer, and UCB Pharma.

\section{References}

[1] Knake S, Rosenow F, Vescovi M et al. Incidence of status epilepticus in adults in Germany: A prospective, population-based study. Epilepsia 2001; 42: 714-718

[2] Delaj L, Novy J, Ryvlin P et al. Refractory and super-refractory status epilepticus in adults: A 9-year cohort study. Acta Neurol Scand 2017; 135: 92-99

[3] Trinka E, Cock H, Hesdorffer D et al. A definition and classification of status epilepticus-Report of the ILAE Task Force on Classification of Status Epilepticus. Epilepsia 2015; 56: 1515-1523

[4] Dobesberger J, Ristic AJ, Walser G et al. Duration of focal complex, secondarily generalized tonic-clonic, and primarily generalized tonic-clonic seizures-A video-EEG analysis. Epilepsy Behav 2015; 49: $111-117$

[5] Shorvon S, Ferlisi M. The treatment of super-refractory status epilepticus: A critical review of available therapies and a clinical treatment protocol. Brain 2011; 134: 2802-2818

[6] Mayer SA, Claassen J, Lokin J et al. Refractory status epilepticus: Frequency, risk factors, and impact on outcome. Arch Neurol 2002; 59: $205-210$

[7] Holtkamp M, Othman J, Buchheim K et al. Predictors and prognosis of refractory status epilepticus treated in a neurological intensive care unit. J Neurol Neurosurg Psychiatry 2005; 76: 534-539

[8] Rossetti AO, Logroscino G, Bromfield EB. Refractory status epilepticus: Effect of treatment aggressiveness on prognosis. Arch Neurol 2005; 62: $1698-1702$

[9] Novy J, Logroscino G, Rossetti AO. Refractory status epilepticus: A prospective observational study. Epilepsia 2010; 51: 251-256

[10] Wilder-Smith EP, Lim EC, Teoh HL et al. The NORSE (new-onset refractory status epilepticus) syndrome: Defining a disease entity. Ann Acad Med Singapore 2005; 34: 417-420

[11] Ferlisi M, Shorvon S. The outcome of therapies in refractory and super-refractory convulsive status epilepticus and recommendations for therapy. Brain 2012; 135: 2314-2328

[12] Loscher W, Brandt C. Prevention or modification of epileptogenesis after brain insults: Experimental approaches and translational research. Pharmacol Rev 2010; 62: 668-700

[13] Claassen J, Hirsch LJ, Emerson RG et al. Treatment of refractory status epilepticus with pentobarbital, propofol, or midazolam: A systematic review. Epilepsia 2002; 43: 146-153

[14] Rossetti AO, Milligan TA, Vulliemoz S et al. A randomized trial for the treatment of refractory status epilepticus. Neurocrit Care 2011; 14: 4-10

[15] Prabhakar H, Bindra A, Singh GP et al. Propofol versus thiopental sodium for the treatment of refractory status epilepticus. Cochrane Database Syst Rev 2012, doi:10.1002/14651858.CD009202.pub2 CD009202

[16] Reznik ME, Berger K, Claassen J. Comparison of Intravenous Anesthetic Agents for the Treatment of Refractory Status Epilepticus. J Clin Med 2016; 5:

[17] Pugin D, Foreman B, De Marchis GM et al. Is pentobarbital safe and efficacious in the treatment of super-refractory status epilepticus: A cohort study. Crit Care 2014; 18: R103

[18] Bellante F, Legros B, Depondt C et al. Midazolam and thiopental for the treatment of refractory status epilepticus: A retrospective comparison of efficacy and safety. J Neurol 2016; 263: 799-806
[19] Walli A, Poulsen TD, Dam M et al. Propofol infusion syndrome in refractory status epilepticus: A case report and topical review. Case Rep Emerg Med 2016: 2016: 3265929

[20] Power KN, Flaatten H, Gilhus NE et al. Propofol treatment in adult refractory status epilepticus. Mortality risk and outcome. Epilepsy Res 2011; 94: 53-60

[21] Morrison G, Gibbons E, Whitehouse WP. High-dose midazolam therapy for refractory status epilepticus in children. Intensive Care Med 2006; 32: 2070-2076

[22] Young GB. Status epilepticus and brain damage: Pathology and pathophysiology. Adv Neurol 2006; 97: 217-220

[23] Rossetti AO, Hurwitz S, Logroscino G et al. Prognosis of status epilepticus: Role of aetiology, age, and consciousness impairment at presentation. J Neurol Neurosurg Psychiatry 2006; 77: 611-615

[24] Marchi NA, Novy J, Faouzi M et al. Status epilepticus: Impact of therapeutic coma on outcome. Crit Care Med 2015; 43: 1003-1009

[25] Sutter R, Marsch S, Fuhr P et al. Anesthetic drugs in status epilepticus: Risk or rescue? A 6-year cohort study. Neurology 2014; 82: 656-664

[26] Sutter R, De Marchis GM, Semmlack $S$ et al. Anesthetics and outcome in status epilepticus: A matched two-center cohort study. CNS Drugs 2017; 31: 65-74

[27] Towne AR, Waterhouse EJ, Boggs JG et al. Prevalence of nonconvulsive status epilepticus in comatose patients. Neurology 2000; 54: 340-345

[28] Claassen J, Mayer SA, Kowalski RG et al. Detection of electrographic seizures with continuous EEG monitoring in critically ill patients. Neurology 2004; 62: 1743-1748

[29] Spatola M, Novy J, Du Pasquier R et al. Status epilepticus of inflammatory etiology: A cohort study. Neurology 2015; 85: 464-470

[30] Gaspard N, Foreman BP, Alvarez V et al. New-onset refractory status epilepticus: Etiology, clinical features, and outcome. Neurology 2015; 85: 1604-1613

[31] Tan RY, Neligan A, Shorvon SD. The uncommon causes of status epilepticus: A systematic review. Epilepsy Res 2010; 91: 111-122

[32] Lowenstein DH, Alldredge BK. Status epilepticus at an urban public hospital in the 1980s. Neurology 1993; 43: 483-488

[33] Borris D], Bertram EH, Kapur J. Ketamine controls prolonged status epilepticus. Epilepsy Res 2000; 42: 117-122

[34] Gaspard N, Foreman B, Judd LM et al. Intravenous ketamine for the treatment of refractory status epilepticus: A retrospective multicenter study. Epilepsia 2013; 54: 1498-1503

[35] Mirsattari SM, Sharpe MD, Young GB. Treatment of refractory status epilepticus with inhalational anesthetic agents isoflurane and desflurane. Arch Neurol 2004; 61: 1254-1259

[36] Vezzani A, Ruegg S. The pivotal role of immunity and inflammatory processes in epilepsy is increasingly recognized: Introduction. Epilepsia 2011; 52: (Suppl 3): 1-4

[37] Zeiler FA, Matuszczak M, Teitelbaum J et al. Plasmapheresis for refractory status epilepticus, part I: A scoping systematic review of the adult literature. Seizure 2016; 43: 14-22

[38] Francois LL, Manel V, Rousselle C et al. Ketogenic regime as anti-epileptic treatment: Its use in 29 epileptic children. Arch Pediatr 2003; 10: $300-306$

[39] Thakur KT, Probasco JC, Hocker SE et al. Ketogenic diet for adults in super-refractory status epilepticus. Neurology 2014; 82: 665-670

[40] Strzelczyk A, Reif PS, Bauer S et al. Intravenous initiation and maintenance of ketogenic diet: Proof of concept in super-refractory status epilepticus. Seizure 2013; 22: 581-583

[41] Legriel S, Lemiale V, Schenck M et al. Hypothermia for Neuroprotection in Convulsive Status Epilepticus. N Engl J Med 2016; 375: 2457-2467 
[42] Niquet J, Gezalian M, Baldwin R et al. Neuroprotective effects of deep hypothermia in refractory status epilepticus. Ann Clin Transl Neurol 2015; 2: 1105-1115

[43] Niquet J, Baldwin R, Gezalian M et al. Deep hypothermia for the treatment of refractory status epilepticus. Epilepsy Behav 2015; 49: 313-317

[44] Lhatoo SD, Alexopoulos AV. The surgical treatment of status epilepticus. Epilepsia 2007; 48 (Suppl 8): 61-65

[45] Lisanby SH, Bazil CW, Resor SR et al. ECT in the treatment of status epilepticus. J ECT 2001; 17: 210-215

[46] Griesemer DA, Kellner CH, Beale MD et al. Electroconvulsive therapy for treatment of intractable seizures. Initial findings in two children. Neurology 1997; 49: 1389-1392

[47] Broomall E, Natale JE, Grimason M et al. Pediatric super-refractory status epilepticus treated with allopregnanolone. Ann Neurol 2014; 76: 911-915

[48] Kokate TG, Cohen AL, Karp E et al. Neuroactive steroids protect against pilocarpine- and kainic acid-induced limbic seizures and status epilepticus in mice. Neuropharmacology 1996; 35: 1049-1056

[49] Reddy K, Reife R, Cole AJ. SGE-102: A novel therapy for refractory status epilepticus. Epilepsia 2013; 54: (Suppl 6): 81-83

[50] Towne AR, Pellock JM, Ko D et al. Determinants of mortality in status epilepticus. Epilepsia 1994; 35: 27-34

[51] Neligan A, Shorvon SD. Prognostic factors, morbidity and mortality in tonic-clonic status epilepticus: A review. Epilepsy Res 2011; 93: 1-10
[52] Rossetti AO, Logroscino G, Bromfield EB. A clinical score for prognosis of status epilepticus in adults. Neurology 2006; 66: 1736-1738

[53] Leitinger M, Holler Y, Kalss G et al. Epidemiology-based mortality score in status epilepticus (EMSE). Neurocrit Care 2015; 22: 273-282

[54] Foreman B, Claassen J, Abou Khaled K et al. Generalized periodic discharges in the critically ill: A case-control study of 200 patients. Neurology 2012; 79: 1951-1960

[55] Rossetti AO, Oddo M. The neuro-ICU patient and electroencephalography paroxysms: If and when to treat. Curr Opin Crit Care 2010; 16: 105-109

[56] Logroscino G, Hesdorffer DC, Cascino G et al. Short-term mortality after a first episode of status epilepticus. Epilepsia 1997; 38: 1344-1349

[57] Kilbride RD, Reynolds AS, Szaflarski JP et al. Clinical outcomes following prolonged refractory status epilepticus (PRSE). Neurocrit Care 2013; 18: 374-385

[58] Cooper AD, Britton JW, Rabinstein AA. Functional and cognitive outcome in prolonged refractory status epilepticus. Arch Neurol 2009; 66: $1505-1509$

[59] Bausell R, Svoronos A, Lennihan L et al. Recovery after severe refractory status epilepticus and 4 months of coma. Neurology 2011; 77: 1494-1495 